\title{
Desempenho de cultivares de mamona no Semiárido paraibano: componentes de crescimento e teor de óleo
}

\section{Performance cultivars castor bean cultivars in Semiarid Paraiba: components of growth and oil content}

\author{
Rodolfo Rodrigo de Almeida Lacerda ${ }^{1}$, Anielson dos Santos Souza ${ }^{2}$, Guilherme de Freitas Frutado ${ }^{3}$, Ivislanne de Sousa \\ Queiroga ${ }^{4}$, Wendell de Almeida Lacerda ${ }^{5}$
}

Resumo: A cultura da mamona vem despontando como uma das principais matérias-primas, para produção de óleo, especialmente no Nordeste, todavia muitos fatores têm dificultado o estabelecimento dessa lavoura em novas áreas dentre os quais destacam-se a falta de cultivares adaptadas a cada região de cultivo. O presente estudo foi desenvolvido com o objetivo de identificar cultivares de mamona com adaptação ao sertão paraibano. O trabalho foi conduzido no Sítio Monte Alegre zona rural do município de Pombal - PB, o delineamento experimental foi em blocos ao acaso com sete tratamentos compostos pelos cultivares BRS Nordestina; BRS Paraguaçu; BRS Energia; AL Guarany-2002; IAC 2028; IAC Guarany e IAC 80, com quatro repetições, foram avaliadas as características de crescimento e teor de óleo nas sementes. Dos resultados registrou-se maior valor de comprimento do racemo as cultivares IAC 80 e IAC 2028, considerando as médias dos racemos até a $3^{\mathrm{a}}$ ordem, para as características de massa e número de frutos por racemos foi constatado que a IAC 80 superou estatisticamente as demais cultivares, o maior teor de óleo nas sementes foi observado na cultivar BRS Energia que também conferiu maior rendimento de óleo entre as sete cultivares. Apesar disso, acredita-se que novos estudos devem ser realizados em diferentes épocas e formas de manejo para se identificar com maior precisão qual cultivar melhor se adapta a região.

Palavras-chave: Ricinus communis L.; Adaptação; Variedades.

Abstract: Currently the culture of castor has emerged as a of the main raw material for the production of oil, especially in the Northeast, however many factors has hampered the establishment of this tilth in new areas among which is the lack of cultivars adapted to each region of cultivation. This study was developed with the objective of identify castor's cultivars with adaptation to the Paraíba Sertão. The study was conducted on Sítio Monte Alegre countryside of municipality of Pombal - PB, the experimental design was the of random blocks with seven treatments composed by BRS Northeastern; Paraguaçu BRS; BRS Energy; Guarany AL-2002, IAC 2028, IAC Guarany and IAC 80, with four replications were evaluated characteristics of growth, production, yield and oil content in the seeds. From the results was registrated a higher From the results recorded was greater value of the length of cluster IAC 80 and IAC cultivars in 2028, considering the averages of racemes up to the third order, the characteristics of mass and number of fruits per raceme was found that 80 IAC statistically outperformed the other cultivars, the higher oil content in seeds was observed in BRS Energy also gave higher oil yield among the seven cultivars. Nevertheless, we believe that further studies should be performed_at different times and forms of management to identify with greater precision farming which best fits the region.

Key words: Ricinus communis L., Adaptation; Varieties.

\footnotetext{
*Autor para correspondência

Recebido para publicação em 20/10/2015; aprovado em 23/12/2015

${ }^{1}$ Eng. Agr. Mestre em Horticultura Tropical, Universidade Federal de Campina Grande, rodolfo-lacerda@hotmail.com

${ }^{2}$ Eng. Agr. Dr. Sc., Professor Adjunto da Unidade Acadêmica de Ciências Agrárias - UFCG/CCTA - Universidade Federal de Campina Grande, Pombal-

PB. E-mail: anielson@ ccta.ufcg.edu.br

${ }^{3}$ Eng. Agr. Mestrado em Engenharia Agrícola, Universidade Federal de Campina Grande, gfreitasagro@gmail.com.

${ }^{4}$ Bióloga especialista em educação ambiental, Universidade Federal de Campina Grande, ivislanne@ hotmail.com

${ }^{5}$ Bacharel em Administração, Universidade Estadual da Paraíba - UFPB, Especialista em auditoria contábil, Faculdade Integradas de Patos - FIP,

wendelllacerda@hotmail.com
} 


\section{INTRODUÇÃO}

A mamoneira (Ricinus communis L.), é uma oleaginosa pertencente à família Euphorbiaceae, destaca-se pelo seu elevado valor socioeconômico, a utilização de seus produtos e coprodutos na indústria ricinoquímica e na agricultura, além da possibilidade, do óleo extraído de suas sementes, ser usado na produção de biocombustível (RIBEIRO et al., 2009). Apesar disso, essa cultura vem passando por sérios problemas em sua cadeia produtiva, relacionados ao uso de cultivares que não apresenta resultados satisfatórios a cada região..

A área cultivada com mamona na safra 2011/12 ficou em torno de 145,0 mil hectares, com redução de 33,9\% em relação à safra anterior. A redução da área cultivada na Bahia chegou a $49,6 \%$, cultivando apenas 71,0 mil hectares, ante os 140,8 mil hectares da safra anterior (CONAB, 2012).

A estiagem verificada na região Nordeste derrubou a produtividade média da safra nacional de mamona, ficando em $504 \mathrm{~kg} / \mathrm{ha}$. E nessa região onde se concentra a maior área cultivada, a produtividade é de $488 \mathrm{~kg} / \mathrm{ha}$. A produção nacional de mamona esperada para esta safra pode alcançar 73 mil toneladas, $48,3 \%$ menor em relação à colheita anterior (CONAB, 2012).

Para o extenso semiárido brasileiro, esta planta é de fácil cultivo, em comparação com outras lavouras, por apresentar certa resistência à escassez de água, o que não garante boas produtividades.

A busca mundial por sustentabilidade ambiental, com base na substituição progressiva dos combustíveis minerais derivados do petróleo por combustíveis renováveis de origem vegetal, dentre eles o biodiesel, criou uma perspectiva real para a expansão do cultivo da mamona em escala comercial (LIRA; BARRETO, 2009).

Apesar de a mamoneira ter sido indicada no início do programa como principal matéria-prima para produção de biodiesel, hoje já se sabe, que devido ao alto valor econômico do óleo de mamona, tal produto tem sido destinado para outras finalidades com maior valor agregado, sendo a demanda atual para produção de biodiesel atendida pela cultura da soja (Glycine max L.). Todavia a cultura da mamona permanece inserida no Programa Nacional de Produção e Uso de Biodiesel (PNPB), e recebe grandes incentivos e investimentos do Governo Federal e da Petrobras.

Existe vários cultivares de mamoneira disponível para o plantio. Eles diferem em porte, deiscência dos frutos, tipo dos cachos, produtividade, entre outras características. Com isso, deve-se verificar entre os vários cultivares existente a que mais se adapta a determinada região (AMARAL, 2008).

É nesse intuito, que cresce a necessidade de se entender o comportamento de cultivares de mamona em relação às mais diversas condições de cultivo, e do ambiente. Fatores esses, que possibilitam a escolha da cultivar que se enquadre melhor com as características presentes no ambiente, de modo, a gerar mais produtividade e lucros para o produtor.

Pelo exposto objetivou-se com o presente estudo avaliar o comportamento de sete cultivares de mamona e dentre elas identificar aqueles que maior apresentam potencial de exploração no sertão paraibano, especificamente no município de Pombal-PB.

\section{MATERIAL E MÉTODOS}

O trabalho foi realizado no período de 27 de abril a 13 de dezembro de 2010 em área situada no Sítio Monte Alegre zona rural do município de Pombal - PB, o qual está situado geograficamente na Microrregião de Sousa, com coordenadas de 184 metros de altitude, $06^{\circ} 46^{\prime}$ de latitude sul e $37^{\circ} 48^{\prime}$ de longitude oeste (BELTRÃO et al., 2005). Segundo Köppen, o clima predominante na região é do tipo Aw: quente e seco com chuvas de verão-outono, precipitações pluviais anuais em torno de $800 \mathrm{~mm}$ e amplitude térmica inferior a $5^{\circ} \mathrm{C}$. $\mathrm{Na}$ área experimental o solo pertence à classe dos NEOSSOLOS Flúvicos (EMBRAPA, 2006).

O delineamento experimental foi o de blocos ao acaso com sete tratamentos compostos pelos cultivares de mamona, a saber, BRS Nordestina; BRS Paraguaçu; BRS Energia; AL Guarany-2002; IAC 2028; IAC Guarany e IAC 80, com quatro repetições, totalizando 28 unidades experimentais com ruas entre blocos e parcelas de 1,0 m. O tamanho da parcela variou com o espaçamento das cultivares, sendo de 2,5 $\mathrm{m}$ para as cultivares de ciclo longo BRS Nordestina, BRS Paraguaçu e IAC 80 e de 1,0 m para as cultivares de ciclo precoce, para as demais, a densidade de semeadura foi de uma planta por metro linear para todas as cultivares. Cada parcela continha três fileiras com oito plantas, sendo tomada como área útil a ocupada pelas quatro plantas localizadas no centro da fileira central, a saber: $10,0 \mathrm{~m}^{2}$ e 4,0 $\mathrm{m}^{2}$, para as cultivares de ciclo longo e precoce, respectivamente. Desse modo, a coleta dos dados foi feita em quatro plantas por parcela e 16 plantas para cada cultivar.

Antes do plantio foram coletadas amostras simples de solo da área experimental para determinar suas características químicas e físicas, bem como para fazer a recomendação de adubação. As quantidades necessárias foram as seguintes: 6040 - $40 \mathrm{~kg} \mathrm{ha}^{-1}$ de NPK, nas formas de ureia, superfosfato simples e cloreto de potássio, respectivamente, conforme a recomendação de adubação e calagem para o estado do Ceará da Universidade Federal do Ceará (1993).

A adubação foi feita em covas, o fósforo e potássio foram aplicados por completo em fundação durante a semeadura. Para o nitrogênio, a aplicação foi parcelada em três partes iguais, sendo, 1/3 em fundação, 1/3 aos 40 dias e $1 / 3$ aos 60 dias após o plantio, em cobertura, onde as cultivares de ciclo longo receberam $12,5 \mathrm{~g}$ e as de ciclo curto $5,0 \mathrm{~g}$ por planta de ureia.

A semeadura ocorreu a partir da abertura das covas manualmente, assim como, a adubação. Semeou-se na profundidade de $5 \mathrm{~cm}$ aproximadamente, três sementes por cova em média. O desbaste foi realizado cortando as plantas rente ao solo 20 dias após a emergência das plântulas, permanecendo uma por cova, resultando em uma população de 4.000 e 10.000 plantas por hectare para as cultivares de ciclo longo e precoce, respectivamente. Quando necessário realizou-se o controle das plantas daninhas através de capinas manuais com auxilio de enxadas

A coleta dos dados foi realizada de acordo com a fenologia de cada cultivar, à medida que os racemos apresentavam-se com pelo menos $70 \%$ dos seus frutos maduros. Os racemos colhidos foram identificados e separados de acordo com a cultivar e a repetição. Em seguida foram colocados para completar a secagem em casa de vegetação por um período de até 20 dias. 
Ao completar a secagem os racemos foram pesados, contados, e separados por repetição e ordem. O beneficiamento foi de forma manual retirando os frutos da raque, os quais foram pesados e contados, para posteriormente serem retiradas as sementes das bagas para pesagem.

Foram coletados os dados referentes a altura de inserção do racemo primário $(\mathrm{cm})$, altura de plantas $(\mathrm{cm})$, diâmetro do caule $(\mathrm{mm})$, número de racemos por planta e número de frutos por racemo, comprimento do racemo $(\mathrm{cm})$ e massa do racemo $(\mathrm{g})$, teor de óleo nas sementes $(\%)$.

Os dados obtidos foram submetidos à análise da variância pelo teste $\mathrm{F}$, e quando verificada significância a $1 \%$ ou $5 \%$ de probabilidade, realizou-se o teste de Tukey ( $\mathrm{p} \leq$ $5 \%$ ) para separação das médias. Todos os procedimentos estatísticos foram realizados com o auxílio do programa computacional para análises estatísticas Saeg V. 9.0 DEMO, da Fundação Arthur Bernardes da Universidade Federal de Viçosa.

Tabela 01. Médias da altura de inserção do racemo primário, altura da planta, e diâmetro do caule de sete cultivares de mamona. Pombal - PB, 2013.

\begin{tabular}{cccc}
\hline CULTIVAR & Altura de inserção $(\mathrm{cm})$ & Altura da planta $(\mathrm{cm})$ & Diâmetro do caule $(\mathrm{mm})$ \\
\hline IAC 2028 & $51,87 \mathrm{c}$ & $94,43 \mathrm{~b}$ & $24,06 \mathrm{~b}$ \\
AL - Guarany 2002 & $59,62 \mathrm{c}$ & $114,81 \mathrm{~b}$ & $26,18 \mathrm{~b}$ \\
BRS Energia & $62,72 \mathrm{c}$ & $129,18 \mathrm{~b}$ & $21,68 \mathrm{~b}$ \\
IAC Guarani & $50,75 \mathrm{c}$ & $91,25 \mathrm{~b}$ & $20,93 \mathrm{~b}$ \\
IAC 80 & $70,31 \mathrm{bc}$ & $105,00 \mathrm{~b}$ & $26,46 \mathrm{~b}$ \\
BRS Paraguaçu & $109,56 \mathrm{ab}$ & $204,37 \mathrm{a}$ & $36,05 \mathrm{a}$ \\
BRS Nordestina & $118,60 \mathrm{a}$ & $188,37 \mathrm{a}$ & $36,65 \mathrm{a}$ \\
\hline D.M.S. & 40,16 & 49,62 & 8,64 \\
\hline
\end{tabular}

Médias seguidas de letras iguais nas colunas não diferem estatisticamente entre si pelo teste de Tukey a $5 \%$ de probabilidade.

média de 33,38 no espaçamento de $2,5 \mathrm{~m}$. Os valores obtidos podem estar relacionados ao fato de estas cultivares serem de
Para a variável altura de planta, as cultivares BRS Paraguaçu e BRS Nordestina, tiveram maiores valores médios, sendo $204,37 \mathrm{~cm}$ e $188,37 \mathrm{~cm}$ respectivamente, não diferenciando estatisticamente (Tabela 1). Segundo Mazzani (1983) a altura de planta é controlada por fatores genéticos e ambientais. Lacerda et. al. (2010), afirma que Altura de plantas e precocidade mantêm estreita relação entre si. A menor média para essa característica foi encontrada na cultivar IAC-Guarani com 91,25 cm, embora não tenha se diferenciado estatisticamente das cultivares IAC 2028, AL Guarany 2002, IAC 80 e BRS Energia.

O fato de a cultivar IAC-Guarani apresentar menor altura de planta e também de inserção do racemo primário pode indicar que ela é de maior precocidade, o que esta coerente com sua descrição. Já para a cultivar IAC 80, considerada de porte alto o comportamento foi de cultivar de porte baixo, mas manteve o ciclo tardio, levando-se a crer, que tal característica está relacionada ao efeito das condições ambientais.

Para os dados de diâmetro do caule, observou-se que a cultivar BRS Nordestina, apresentou maior diâmetro $(36,65)$ em relação a maioria das cultivares, a exceção foi a cultivar BRS Paraguaçu não diferenciando estatisticamente (Tabela 1). Resultado superior ao relatado por Severino et al. (2006), quando avaliando diferentes espaçamento para a BRS Nordestina no município de Quixeramobim, CE, obtendo

\section{RESULTADOS E DISCUSSÃO}

Com relação à altura de inserção do racemo primário, observou-se que a BRS Nordestina foi a que apresentou o maior valor $(118,60 \mathrm{~cm})$ em relação às demais (Tabela 1). Tal resultado é coerente tendo em vista que a BRS Nordestina é mais tardia e também possui maior porte. Ferreira, Maruyama e Soratto (2009) também constataram que a BRS Nordestina emite o racemo primário a uma maior altura que plantas de porte baixo como os híbridos Lyra, Savana e a BRS Energia. Por outro lado, o menor valor foi encontrado na cultivar IAC Guarani $(50,75 \mathrm{~cm})$, sem diferença estatística em relação as cultivares IAC 2028, AL - Guarani 2002 e BRS Energia, o que é coerente já que estas cultivares são mais precoces e de menor porte. Tais resultados corroboram com informações de Beltrão (2003), ao citar que a altura do primeiro racemo é uma característica ligada a precocidade da planta, sendo considerada mais precoce a planta que lança o primeiro racemo em menor altura, (SOUZA, 2007) também verificaram menor altura de inserção do racemo primário para as cultivares Mirante 10 e BRS Energia, respectivamente em relação a cultivar BRS Nordestina. em campo o que favorece maior expansão do caule. Com tais resultados, fica evidente que as cultivares BRS Nordestina e BRS Paraguaçu possuem maior crescimento que as demais.

A cultivar IAC 80 embora seja de ciclo longo apresentou um diâmetro caulinar de 26,46 mm que é o menor valor quando comparado as demais cultivares de ciclo longo o que se deve possivelmente ao efeito das condições edafoclimáticas da região semiárida, sendo inclusive observado alteração no ciclo desta cultivar. A menor média apresentada para essa característica foi da cultivar IAC Guarani. Cumpre informar que com exceção dos dados do diâmetro da IAC 80, os demais são coerentes, pois cultivares de maior porte necessitam de maiores diâmetros já que a planta possui maior estrutura, inclusive com a emissão de um maior número de ramos laterais.

As cultivares IAC 80 e IAC 2028 apresentaram maiores valores de comprimento do racemo com 43,50 cm e 40,28 $\mathrm{cm}$, respectivamente, considerando as médias dos racemos até a $3^{\mathrm{a}}$ ordem (Tabela 2 ), entre as duas sobressai em importância o fato de a IAC 2028 possuir maior precocidade, o que na prática significa maiores racemos em menor tempo. O menor comprimento efetivo médio, entre as cultivares estudas foi observado na BRS Paraguaçu com 14,43 cm, talvez por ser uma característica da própria cultivar que possui racemos ovais, também é possível que mamoneiras com racemos 
curtos compensem tal característica com a emissão de mais racemos por planta.

Com relação à massa do racemo, a cultivar IAC 80 apresentou um maior valor correspondendo a 62,22 $\mathrm{g}$ em média considerando as três primeiras ordens, diferindo estatisticamente das demais cultivares, este valor esta relacionado ao maior número de frutos por racemo obtido pela cultivar sendo superior as demais com cerca de 74,49 frutos em média por racemo. A menor massa do racemo foi observada na cultivar BRS Energia e BRS Paraguaçu com 13,63 g e 28,04 g, respectivamente (Tabela 2). Para a BRS Paraguaçu o baixo valor de massa do racemo pode estar relacionado ao menor comprimento dos seus racemos (Tabela 2).

Tabela 2. Médias do comprimento e massa dos racemos e número de frutos por racemos de sete cultivares de mamona. Pombal - PB, 2013.

\begin{tabular}{|c|c|c|c|c|}
\hline CULTIVAR & $\begin{array}{l}\text { Comprimento do } \\
\text { racemo }(\mathrm{cm})\end{array}$ & $\begin{array}{c}\text { Massa do racemo } \\
(\mathrm{g}) *\end{array}$ & $\begin{array}{l}\text { Número de frutos por } \\
\text { racemo (unid) }\end{array}$ & $\begin{array}{l}\mathrm{N}^{\circ} \text {. de racemo } \\
\text { planta (unid) }\end{array}$ \\
\hline IAC 2028 & $40,28 \mathrm{a}$ & $32,24 \mathrm{~b}$ & $53,70 \mathrm{~b}$ & $5,06 \mathrm{~b}$ \\
\hline AL - Guarany 2002 & $35,62 \mathrm{ab}$ & $30,57 \mathrm{~b}$ & $43,03 \mathrm{bc}$ & $5,81 \mathrm{~b}$ \\
\hline BRS Energia & $24,00 \mathrm{bc}$ & $13,63 \mathrm{c}$ & $32,62 \mathrm{c}$ & $11,43 \mathrm{a}$ \\
\hline IAC Guarani & $28,50 \mathrm{abc}$ & $29,77 \mathrm{~b}$ & $37,19 \mathrm{bc}$ & $4,18 \mathrm{~b}$ \\
\hline IAC 80 & $43,50 \mathrm{a}$ & $62,22 \mathrm{a}$ & 74,49 a & $4,91 \mathrm{~b}$ \\
\hline BRS Paraguaçu & $14,43 \mathrm{c}$ & $28,04 \mathrm{bc}$ & $27,33 \mathrm{c}$ & $11,62 \mathrm{a}$ \\
\hline BRS Nordestina & $31,18 \mathrm{ab}$ & $34,13 \mathrm{~b}$ & $39,29 \mathrm{bc}$ & $10,62 \mathrm{a}$ \\
\hline D.M.S. & 16,67 & 15,93 & 20,39 & 4,14 \\
\hline
\end{tabular}

Médias seguidas de letras iguais nas colunas não diferem estatisticamente entre si pelo teste de Tukey a 5\% de probabilidade. * Média de quatro repetições e dos racemos até terceira ordem.

Para a característica número de frutos por racemos foi constatado que a IAC 80 superou estatisticamente as demais cultivares com 74,49 frutos por racemo, esse maior número de frutos pode ter propiciado maior massa do racemo, e também pode estar relacionado ao maior comprimento do racemo de tal cultivar, e a maior emissão de flores femininas. Uma vez que (KOUTROUBAS; PAPAKOSTA; DOITSINIS, 1999) salientam que o número de frutos por racemo depende primordialmente do número de flores femininas, levando-se a crer que a cultivar IAC 80 também possui racemos com maior número de flores femininas. Já a cultivar BRS Paraguaçu obteve o menor valor para esta característica, com 27,33 frutos por racemo em média (Tabela 02) podendo estar relacionado as características genéticas da cultivar.

O maior número de racemos por planta foi observado nas cultivares BRS Paraguaçu, BRS Energia e BRS Nordestina com $11,62,11,43$ e 10,62, respectivamente, diferindo estatisticamente das demais cultivares (Tabela 2). Esses valores são superiores as médias descritas por Beltrão et al. (2003) segundo o qual as médias para a BRS Nordestina são de 5 a 7 racemos por planta, e para a BRS Energia segundo a (EMBRAPA, 2007) é de 8 por planta em maiores espaçamentos. Os menores valores para o número de racemo por planta foram observados nas cultivares IAC Guarani e IAC 80 , apresentando 4,18 e 4,91 racemos por planta, respectivamente, podendo este baixo número estar relacionado a não adaptação das cultivares as condições edafoclimáticas da região, a cultivar IAC 80 foi desenvolvida para climas mais amenos como da região sudeste, o que pode ter impossibilitado que a cultivar conclui-se seu ciclo que é de 240 dias, reduzindo assim o número de racemos por planta conforme salienta Zuchi (2008).

As maiores massas de cem sementes foram observadas na cultivares BRS Paraguaçu e BRS Nordestina com médias de 55,48 e 48,45g, sendo superiores estatisticamente as demais cultivares (Tabela 3). Apesar disso, tais valores são inferiores aos reportados na literatura para estas duas cultivares que é de 68 e $71 \mathrm{~g}$ respectivamente, inferindo-se que as condições de cultivo e ambientais podem ter contribuído com tal evento. Dentre as cultivares estudadas a que teve a menor massa de sementes foi a IAC 2028, também com valor abaixo do mencionado na literatura para tal cultivar que é de $45 \mathrm{~g}$ (Tabela 3).

Com relação ao teor de óleo (Tabela 3), a cultivar BRS Energia $(55,14 \%)$ superou estatisticamente as cultivares IAC 2028 e IAC 80. Por outro lado o menor valor foi registrado na cultivar IAC 2028, considerando amostras retiradas de sementes de racemos de $1^{\mathrm{a}}$ a $3^{\mathrm{a}}$ ordens. (KOUTROUBAS; PAPAKOSTA; DOITSINIS, 2000) afirmam que as condições ambientais interferem decisivamente no teor de óleo da semente, especialmente temperatura e disponibilidade de umidade, o que pode ter ocorrido com a cultivar, tendo em vista, que essa cultivar tem baixa adaptação para o semiárido nordestino.

Tabela 3. Médias da massa de cem sementes e teor de óleo das sementes de sete cultivares de mamona. Pombal - PB, 2013.

\begin{tabular}{ccc}
\hline CULTIVAR & Massa de 100 sementes $(\mathrm{g})$ & Teor de óleo $(\%)$ \\
\hline IAC 2028 & $23,33 \mathrm{~b}$ & $48,41 \mathrm{~b}$ \\
AL - Guarany 2002 & $28,91 \mathrm{~b}$ & $51,47 \mathrm{ab}$ \\
BRS Energia & $26,24 \mathrm{~b}$ & $55,14 \mathrm{a}$ \\
IAC Guarani & $26,68 \mathrm{~b}$ & $51,59 \mathrm{ab}$ \\
IAC 80 & $30,35 \mathrm{~b}$ & $49,12 \mathrm{~b}$ \\
BRS Paraguaçu & $55,48 \mathrm{a}$ & $51,30 \mathrm{ab}$ \\
BRS Nordestina & $48,45 \mathrm{a}$ & $53,37 \mathrm{ab}$ \\
\hline D.M.S. & 7,83 & 5,98
\end{tabular}

Médias seguidas de letras iguais nas colunas não diferem estatisticamente entre si pelo teste de Tukey a 5\% de probabilidade. 


\section{CONCLUSÕES}

As cultivares IAC 80 e IAC 2028 apresentam maiores valores de comprimento do racemo dos racemos;

Cultivar IAC 80 possui racemos mais pesados e com maior número de frutos por racemo;

A cultivar BRS Energia possui maior teor de óleo nas sementes o que lhe confere maior rendimento de óleo por hectare;

Acredita-se que novos estudos devem ser realizados em diferentes épocas e formas de manejo, para se indicar com melhor precisão a qual a cultivar que se adapta melhor a região.

\section{REFERÊNCIAS}

AMARAL, J. G. C. do. Mamona al Guarany - 2002. Disponível. Em http//www.cati.sp.gov.br/Cati. Acessado em Mai. 2008.

BELTRÃO, N. E. M.; MELO, F. de B.; CARDOSO, G. D. e SEVERINO, L. S.. Mamona: Árvore do Conhecimento e Sistemas de Produção para o Semi-Árido Brasileiro. Campina Grande, Embrapa Algodão, set., 2003, 19p. (Embrapa Algodão. Circular Técnica, 70).

BELTRÃO, B.A. et al. Diagnóstico do município de Pombal. Projeto cadastro de fontes de abastecimento por água subterrânea. Ministério de Minas e Energia/CPRM/PRODEM. Recife, 2005. 23p.

\section{CONAB COMPANHIA NACIONAL}

$\mathrm{DE}$

ABASTECIMENTO. Acompanhamento da Safra Brasileira de Grãos 2011/12 - Oitavo Levantamento - Maio/2012. Disponívelem:<http://www.conab.gov.br/OlalaCMS/uploads/ arquivos/12_05_10_08_49_52_boletim_maio_2012.pdf>.

Acesso em 06 de fevereiro 2013.

EMBRAPA. Centro Nacional de Pesquisa de Solos. Sistema Brasileiro de Classificação de Solos. 2 ed. Rio de Janeiro, 2006. 212p.

FERREIRA, M. G. C.; MARUYAMA, W. I.; SORATTO R. P. Avaliação de cultivares de mamona em dois arranjos de plantas no outono-inverno em Cassilândia-MS. Rev. bras. ol. fibros., Campina Grande, v.13, n.2, p.53-60, maio/ago. 2009.

KOUTROUBAS, $\quad$ S. D.; PAPAKOSTA, D. K.; DOITSINIS,A. Water requirements for castor oil crops (Ricinus communis L.), in a Mediterranean climate. Agronomy Journal and Crop Science, v. 184, n. 01, p. 33-41, 2000.

KOUTROUBAS, S. D.; PAPAKOSTA, D. K.; DOITSINIS, A. Adaptation and yielding ability of castor plant (Ricinus communis L.) genotypes in a Mediterranean climate. European Journal of Agronomy, v. 11, p. 227-237, 1999.

LACERDA, R. R. de A.; SOUZA, A. dos S.; TÁVORA, F. J. A. F; BELTRÃO, N. E. de M; FURTADO G. de F.; SOUSA, J. R. M.; SOUSA, J. R. de J. Estudo de correlação entre características agronômicas da mamoneira. In: VI Congresso Brasileiro de Mamona e I Simpósio Internacional de Oleaginosas Energéticas. Anais..., João Pessoa, PB - 2010.
LIRA, M. A.; BARRETO, F. P. Oleaginosas com fonte de matéria-prima para a produção de biodiesel. 1.ed. Natal: EMPARN, 2009. 64p.

MAZZANI, B. Euforbiáceas oleaginosas. Tártago. In: MAZZANI, B. Cultivo y mejoramento de plantas oleaginosas. Caracas: Fondo Nacional de Investigaciones Agropécuarias, 1983. p. 227-360.

RIBEIRO, S.; CHAVES, L. H. G.; GUERRA, H. O. C. GHEYI, H. R.; LACERDA, R. D. Resposta da mamoneira cultivar BRS-188 Paraguaçu à aplicação de nitrogênio, fósforo e potássio. Revista Ciência Agronômica. Fortaleza, v.40, n. 4, p. 465-473, out-dez, 2009.

SEVERINO, L. S.; MILANI, M.; MORAES, C. R. de A.; GONDIM, T. M. de S. e CARDOSO, G. D.. Avaliação da produtividade e teor de óleo de dez genótipos de mamoneira cultivados em altitude inferior a 300 metros. Revista Ciência Agronômica, v.37, n.2, p.188-194, 2006.

SOUZA, A. dos S. Manejo Cultural da Mamoneira: Época de Plantio, Irrigação, Espaçamento e Competição de Cultivares. Fortaleza - CE, 2007. 212p. Tese (Doutor em Agronomia), Universidade Federal do Ceará.

UNIVERSIDADE FEDERAL DO CEARÁ. Recomendações de adubação e calagem para o Estado do Ceará. Fortaleza: UFC/CCA, 1993. 248p.

ZUCHI, J. Características Agronômicas de Cultivares de Mamona em Função do Ambiente de Cultivo. Pelotas - RS, 2008. 54p. Dissertação (Mestre em Ciências), Universidade Federal de Pelotas. 